\title{
Molecular dynamics studies on Mdm2 complexes: An analysis of the inhibitor influence
}

\author{
Anna Maria Almerico*, Marco Tutone, Licia Pantano, Antonino Lauria \\ Dipartimento di Scienze e Tecnologie Molecolari e Biomolecolari, Sezione di Chimica Farmaceutica e Biologica, Università di Palermo, Via Archirafi 32,90123 Palermo, Italy
}

\section{A R T I C L E I N F O}

Article history:

Received 13 June 2012

Available online 3 July 2012

\section{Keywords:}

Mdm2

p53

Nutlin

Benzodiazepine

Molecular dynamics

\begin{abstract}
A B S T R A C T
p53 is a powerful anti-tumoral molecule frequently inactivated by mutations or deletions in cancer. However, half of all human tumors expresses wild-type p53, and its activation, by antagonizing its negative regulator Mdm2, might offer a new strategy for therapeutic protocol. In this work, we present a molecular dynamics study on Mdm2 structure bound to two different known inhibitors with the aim to investigate the structural transitions between apo-Mdm2 and Mdm2-inhibitor complexes. We tried to gain information about conformational changes binding a benzodiazepine derivative inhibitor with respect the known nutlin and the apo form. The conformational changes alter the size of the cleft and were mainly in the linker regions, suggesting that the overall dynamic nature of Mdm2 is related to dynamic movements in these regions.
\end{abstract}

(c) 2012 Elsevier Inc. All rights reserved.

\section{Introduction}

In response to DNA damage and other types of stress stimuli, p53 causes cell-cycle arrest [1] or activates apoptosis [2,3]. In normal cells, p53 is held in check until needed by Mdm2 [4]. Harmful mutations of p53 are common mechanisms for the loss of p53 wild-type activity in tumor cells [5]. But another important mechanism is overexpression of Mdm2, which leads to constitutive inhibition of $\mathrm{p} 53$; this is commonly seen in cancerous cells containing wild-type p53 [6,7]. Because of its importance in cancer development, the p53-Mdm2 complex is a really interesting target for anticancer drug design. It has been shown that a p53 homologue is sufficient to induce p53-dependent cell death in cells overexpressing Mdm2 [8], and that a peptide as short as six residues could bind to Mdm2 in the same manner [9]. Medicinal chemistry modifications to the same 6-residue peptide dramatically increased its inhibitory activity [10]. Small p53 mimics would be expected to disrupt the p53-Mdm2 complex. This anti-Mdm2 approach has been shown to re-establish p53 activities in malignant tissues with amplified Mdm2 genes [11]. An increasing number of small-molecule p53-Mdm2 binding inhibitor has been discovered in recent years, such as nutlins, but only few compounds have acceptable cellular potency and selectivity for their molecular target and might represent viable leads for development of therapeutic agents [11].

Abbreviations: MD, molecular dynamics; IMZ, nutlin2; BDZ, benzodiazepine IFD, induced fit docking.

* Corresponding author. Fax: +39 09123860854.

E-mail address: annamaria.almerico@unipa.it (A.M. Almerico).
In order to design an effective p53 mimic as inhibitor of human Mdm2, it is important to understand the p53-Mdm2 interaction at the atomic level. Recently, in different molecular dynamics (MD) studies, the p53-Mdm2 system was investigated to explore the binding interface, and the effect of mutating key residues in the human p53-Mdm2 complex [12-16]. For example, Carlson and Zhong [13] have applied a $2 \mathrm{~ns}$ MD simulation to examine the binding interface in the human p53-Mdm2 complex in order to design a potent p53 mimic, which suggested that an additional hydrofobic pocket should possibly be used to design new Mdm2-inhibitors. More recently Verma et al. [17] used MD simulations to investigate the binding of p53 and nutlin to Mdm2 and MdmX. Simulations reveal that p53 has a higher affinity for Mdm2 than MdmX, driven by stronger electrostatic interactions. The differences are more pronounced for nutlin because this is a small molecule whose binding is driven by short range van der Waals interactions and lacks the long range electrostatics that mediate interactions with p53. In a work carried out on the Xray structure of MdmX bound to a single-domain antibody, Fersht et al. [18] studied structural changes on removal of the ligands: the binding pocket converged to a common conformation, indicating that the differences are due to induced fit. However, the residues that comprise the Mdm2 lid are not conserved in MdmX; and also crystal structure of nutlin2 complexed to Mdm2 (PDB ID: 1RV1) used for the simulation does not contain information concerning the lid. All these studies demonstrated as Mdm2 interconverts between different states in relation to the presence of p53 [19].

In this work we tried to gain information by means MD simulations of Mdm2, complexed with a benzodiazepine derivative and 
nutlin in presence of the lid, with the aim to investigate the structural transitions between apo-Mdm2 and Mdm2-inhibitor bound forms. Moreover the behavior in presence of p53 and small-molecules inhibitors was evaluated. Local and global dynamics are discussed and their implication for drug design addressed.

\section{Materials and methods}

The GROMACS package $[20,21]$ was used to perform molecular dynamics simulations, using the force-field parameter set 43A1. The ligand was removed from the protein and 35 ns MD simulations of the free receptor were first performed, then 35 ns MD simulations of the Mdm2 complexed with the inhibitors were carried out. The systems were embedded in a water box, the simple point charge (SPC) water model, with margin of $9 \AA$ A between the protein and the boundaries of the periodic box. Chlorine counterions were added to produce a neutral charge on the system, and the simulations were performed in constant NPT ensemble. Lennard-Jones potentials were used to model the guest-host and guest-guest interactions, force calculations were truncated at a distance of $1.4 \mathrm{~nm}$. For the calculation of long-range electrostatic forces, the particle-mesh Ewald (PME) method was used, with coulomb cutoff set to $0.9 \mathrm{~nm}$. The temperature was fixed at $300 \mathrm{~K}$ using the Berendsen thermostat and the Berendsen pressure coupling algorithm was used to keep the pressure constant at 300 bar. A steepest-descent energy minimization of the systems was first performed to relax the solute-solvent contacts and in order to remove bad van der Waals contacts. The second step consisted in position-restrained $\mathrm{MD}$, restraining the atom positions of the macromolecule while letting the solvent move in the simulation, to soak the water molecule into the protein. Finally, the third step consisted of the MD simulation. The trajectory files were analyzed by using GROMACS utilities. Moreover VEGA [22] and VMD [23] programs were employed for trajectory analysis and to manipulate the simulation snapshot structures.

The Induced Fit Protocol (IFD), developed by Schrödinger [24], is a method for modeling the conformational changes induced by ligand binding. This protocol models induced fit docking of one or more ligands using the following steps:

1. Constrained minimization of the receptor with an RMSD cutoff of $0.18 \AA$.

2. Initial glide docking of each ligand using a softened potential (van der Waals radii scaling).

3. One round of prime side-chain prediction for each protein/ ligand complex, on residues within a given distance of any ligand pose $(5 \AA)$.

4. Prime minimization of the same set of residues and the ligand for each protein/ligand complex pose. The receptor structure in each pose now reflects an induced fit to the ligand structure and conformation.

5. Glide redocking of each protein/ligand complex structure within a specified energy of the lowest-energy structure. The ligand is now rigorously docked, using XP Glide, into the induced-fit receptor structure.

6. Estimation of the binding energy (IFDScore) for each output pose.

Each complex was then ranked according to the IFD score.

\section{Results and discussion}

The conformational evolution of apo-Mdm2 and bound $\mathrm{Mdm} 2$ was investigated by $35 \mathrm{~ns}$ simulations. Crystal structure of the Mdm2 complexed with the benzodiazepine derivative BDZ (PDB
ID: 1T4E) was used as starting model for simulations. The protein comprises a minimal N-terminal p53 binding domain (residues 16-25), referred to as the lid. While structures of Mdm2 bound to various inhibitors have been solved by X-ray crystallography and NMR [25], no information is available concerning the lid behavior in apo-Mdm2. The limited amount of experimental data thereby precluded the accurate modeling of the lid and its precise arrangement with respect to the cleft. In addition, it has been proposed that the binding event induces global conformational changes of Mdm2 [26].

Here, we report the characterization of the behavior of Mdm2, including the lid, in the apo state (removing ligands), and in complex with the inhibitors. Further by using the obtained apo state at 0 ns, a different inhibitor, IMZ (nutlin2), was docked and multiple poses of the ligand complex were generated with Induced Fit Docking [24]. This model reproduced a conformation nearly identical to the native pose of IMZ as found in $1 \mathrm{RV} 1$, in which the lid was not solved, and that can be used in $35 \mathrm{~ns}$ MD simulation.

The structural changes resulting from the simulation can be, first, evaluated in terms of RMSD of the C $\alpha$-atoms of the bound (Mdm2 + BDZ, Mdm2 + IMZ) and unbound (apo) systems (Fig. 1A).

The trajectory of unbound system maintains quite stable up to about $10 \mathrm{~ns}$ and then increases by about $2 \AA$. After 20 ns increases again, then it remains stable throughout the following $15 \mathrm{~ns}$ of the simulation, with fluctuation of about $0.5 \AA$. When the RMSD of the apo form is compared to that of the Mdm2 bound to IMZ, it was observed that during the first 3.3 ns RMSD was similar and comparable. Thereafter it showed a significant increase, indicating major structural deviations up to $11.3 \mathrm{~ns}$. During the rest of the simulation the dynamics of Mdm2 bound to IMZ remains very stable and was very similar to apo-Mdm2. The RMSD of the Mdm2 bound to BDZ appears different from the previous ones, and showed a significant increase during the first $6 \mathrm{~ns}$ of simulation. After that point the dynamics of the protein remained relatively stable, with fluctuation of about $0.5-0.6 \AA$. In this range, RMSD was comparable with that observed in the Mdm2 bound to IMZ. However, during the rest of the simulation, it showed a remarkable increase of the calculated value, therefore major conformational changes on the protein take place.

Similar behavior of apo-Mdm2 was found in other studies $[13,27]$. The trajectory of the p53-Mdm2 complex [13] was very similar to that observed for Mdm2 bound to IMZ. p53-Mdm2 complexes were highly flexible in simulation, and the complexes examined by us appear to follow this trend.

The RMSD of backbone atoms in the binding pocket (Leu54, Leu57, Ile61, Met62, Tyr67, Val75, Phe86, Phe91, Val93, Ile99, and Ile103) is presented in Fig. 1B. In apo-Mdm2, the binding pocket was stable throughout the trajectory with fluctuation below 0.5 . Binding pocket dynamics of Mdm2 bound to IMZ was very similar to apo-Mdm2. Instead in presence of BDZ, the RMSD gradually increased.

When the RMSD of the lid is compared for apo and bound Mdm2 (Fig. 1C), it was observed that IMZ induced changes in the lid conformation. The trajectory of lid in the apo Mdm2 is quite stable over the timescale of simulation, whereas the RMSD of Mdm2-IMZ lid was $2 \AA$ during the first $5 \mathrm{~ns}$, but an increase of about $2 \AA$ was observed in subsequent ns. A rise in RMSD was observed for Mdm2-BDZ lid between 1 and 24 ns. During the rest of the simulation RMSD showed a significant decrease, suggesting the probable interaction between BDZ and the lid that helps to stabilize lid conformation.

These results indicates the structural stability of apo protein, while the RMSDs of bound systems suggested structural mobility. The presence of inhibitors in the binding pocket caused a different shift of the lid, originally located above the binding cleft. While IMZ seems to push the lid away from the cleft, BDZ allows, in the last 

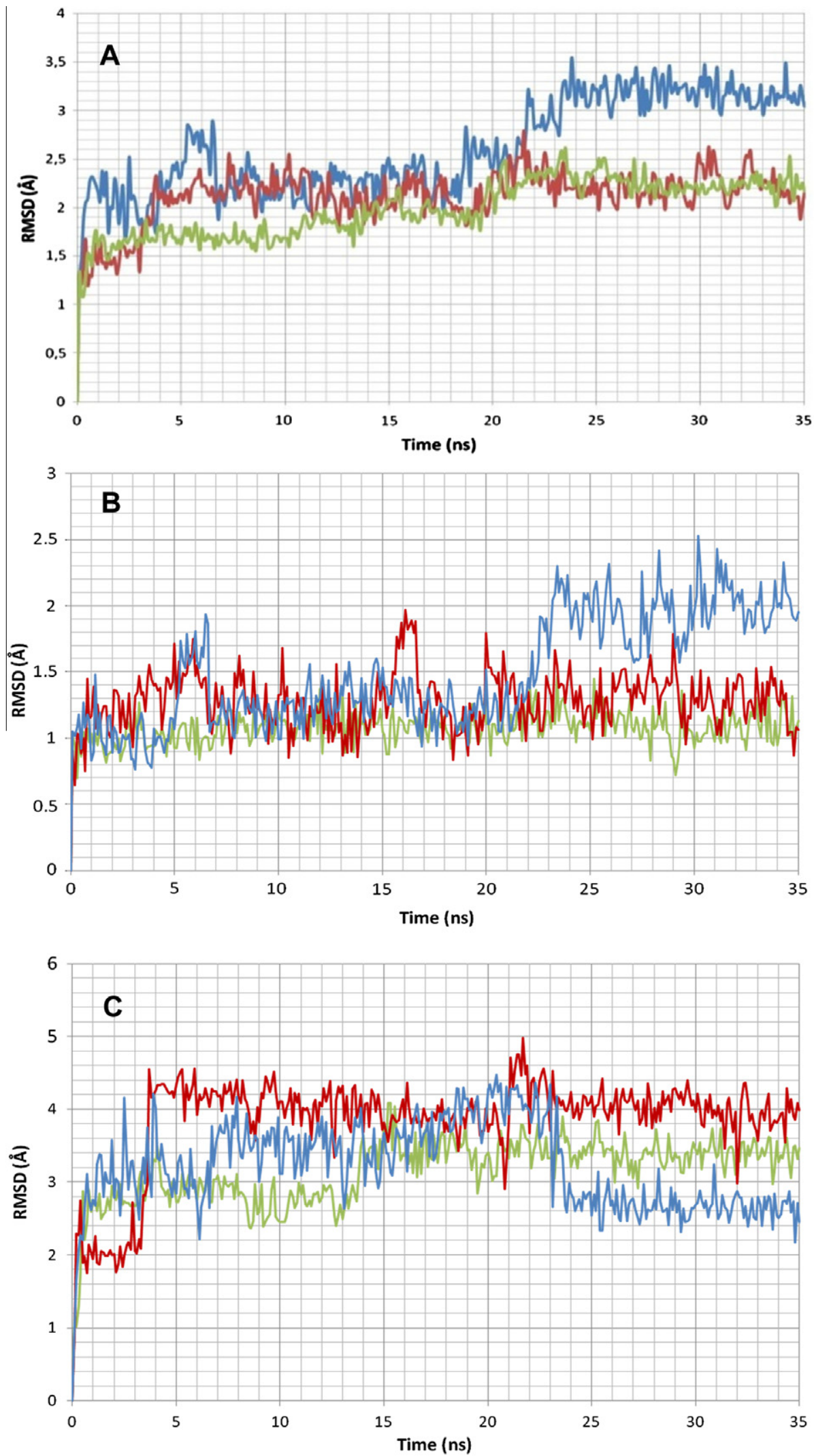

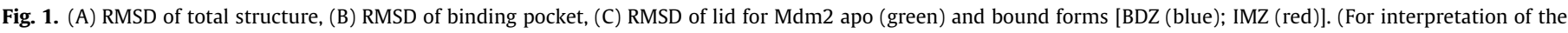
references to color in this figure legend, the reader is referred to the web version of this article.)

section of the simulation, the re-approaching of the lid. Also the movement of binding cleft is adaptive and the inhibitors induce flexibility at this point.
We used PCA as a guide to identify significant dynamic processes from this very large data set. The first step in PCA is the construction of the covariance matrix, which captures the degree of 


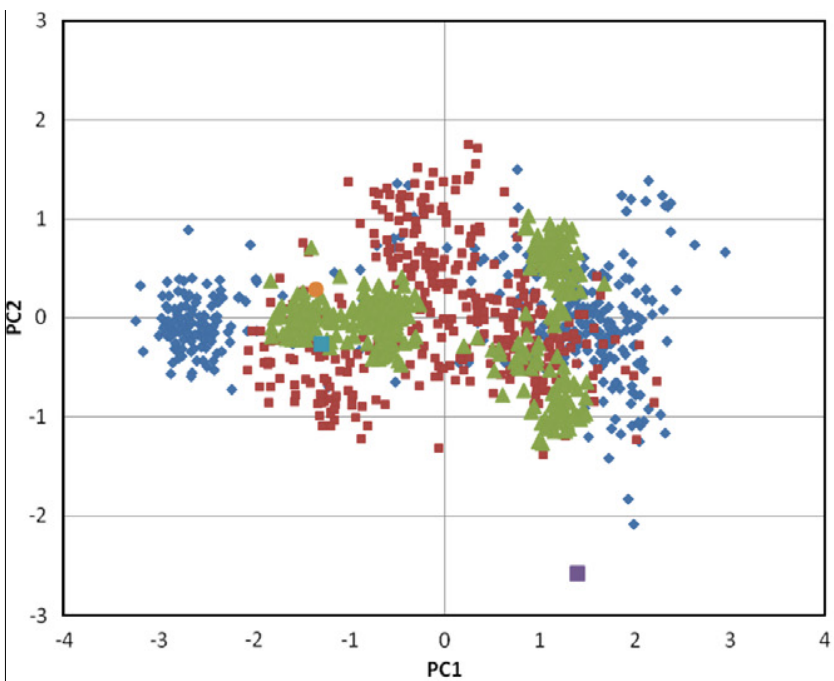

A
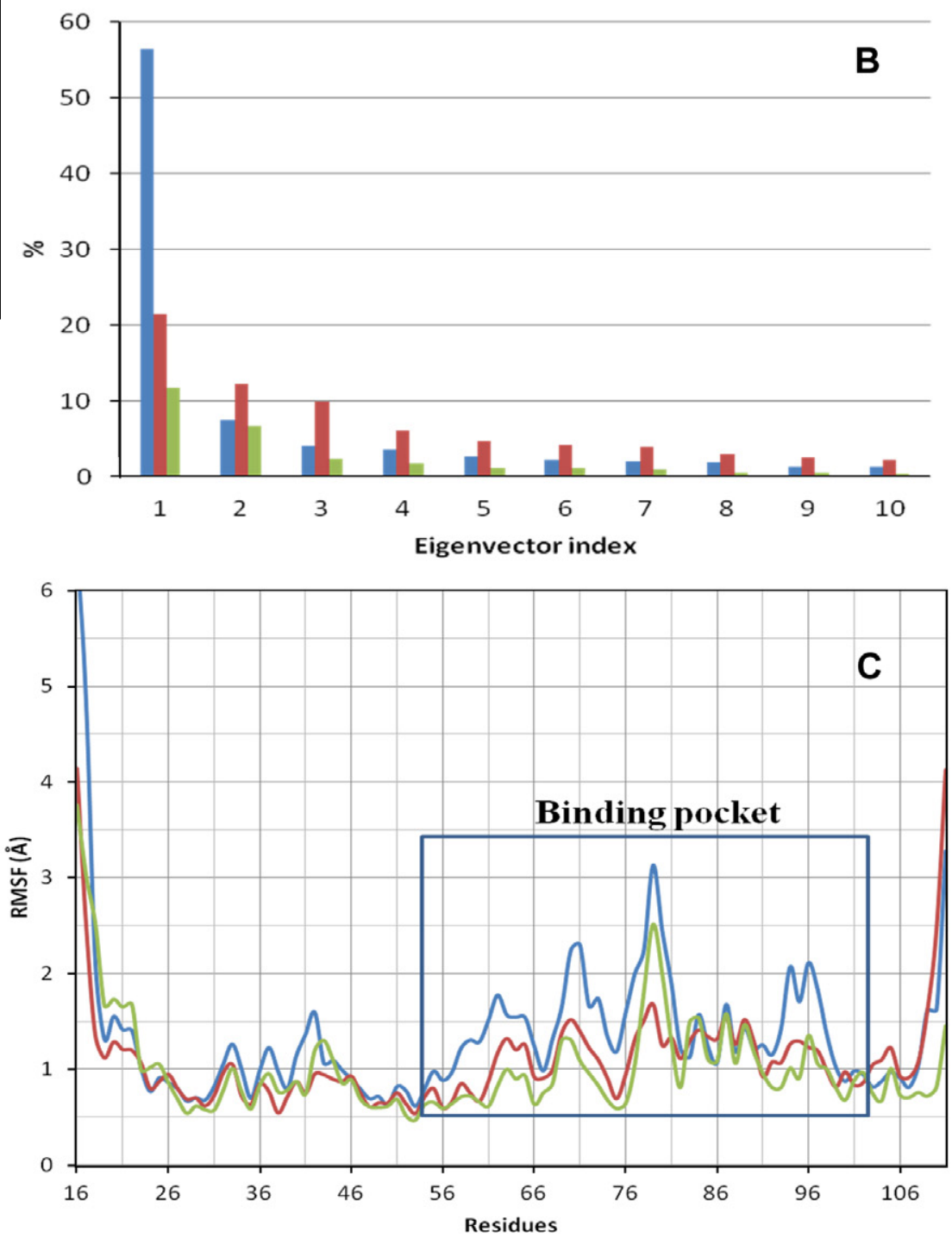

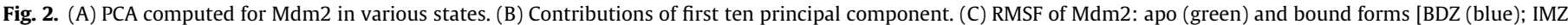
(red)]. (For interpretation of the references to color in this figure legend, the reader is referred to the web version of this article.)

collinearity of atomic motions for each pair of atoms. The covariance matrix is subsequently diagonalized, yielding a matrix of eigenvectors and a diagonal matrix of eigenvalues. Each of the eigenvectors describes a collective motion of particles, where the values of the vector indicate how much the corresponding atom participates in the motion. The sum of the eigenvalues is a measure 
of the total motility in the system. Compared to bound proteins $\left(7.07 \mathrm{~nm}^{2}\right.$ in the case of Mdm2-BDZ, $4.25 \mathrm{~nm}^{2}$ for Mdm2-IMZ) the total sum of eigenvalues of apo system was relatively small $\left(3.53 \mathrm{~nm}^{2}\right)$, indicating a more rigid structure. PCA technique decomposes the intrinsic flexibility of a protein into motions of different frequencies of vibrations. These are then ordered such that the first component (PC1) characterizes the motion with the largest amplitude and lowest frequency.

Principal components were computed for $\mathrm{Mdm} 2$ in various states: apo, complexes with IMZ and with BDZ (Fig. 2A). PC1 dominates the motions of Mdm2 bound to BDZ (covering 56\% of overall motion). The motion is more distributed across PC1 and PC2 in Mdm2 bound to IMZ (21.5\% PC1, 12.2\% PC2) and apo-Mdm2
(11.7\% PC1, 6\% PC2). Histogram in Fig. 2B refers to the contributions of first ten individual principal component to the overall fluctuations. The distribution of the structures in the phase space defined by PC1 and PC2 showed that bound states of the protein with both inhibitors are more flexible than apo one.

To quantitatively measure the mean backbone mobility for each residue, the root mean square fluctuations (RMSF) relative the average structure of apo-Mdm2 were calculated (Fig. 2C). It was observed that the mobility of the unbound system was relatively low for the regions including residues 26-76. In this region the RMSF was < $1 \AA$. Few residues (42-44 and 69-71) displayed a mobility higher than $1 \AA$ A, specifically, residues 69-71 which form a hinge that connects helix $\alpha 2$ with the region formed by sheets
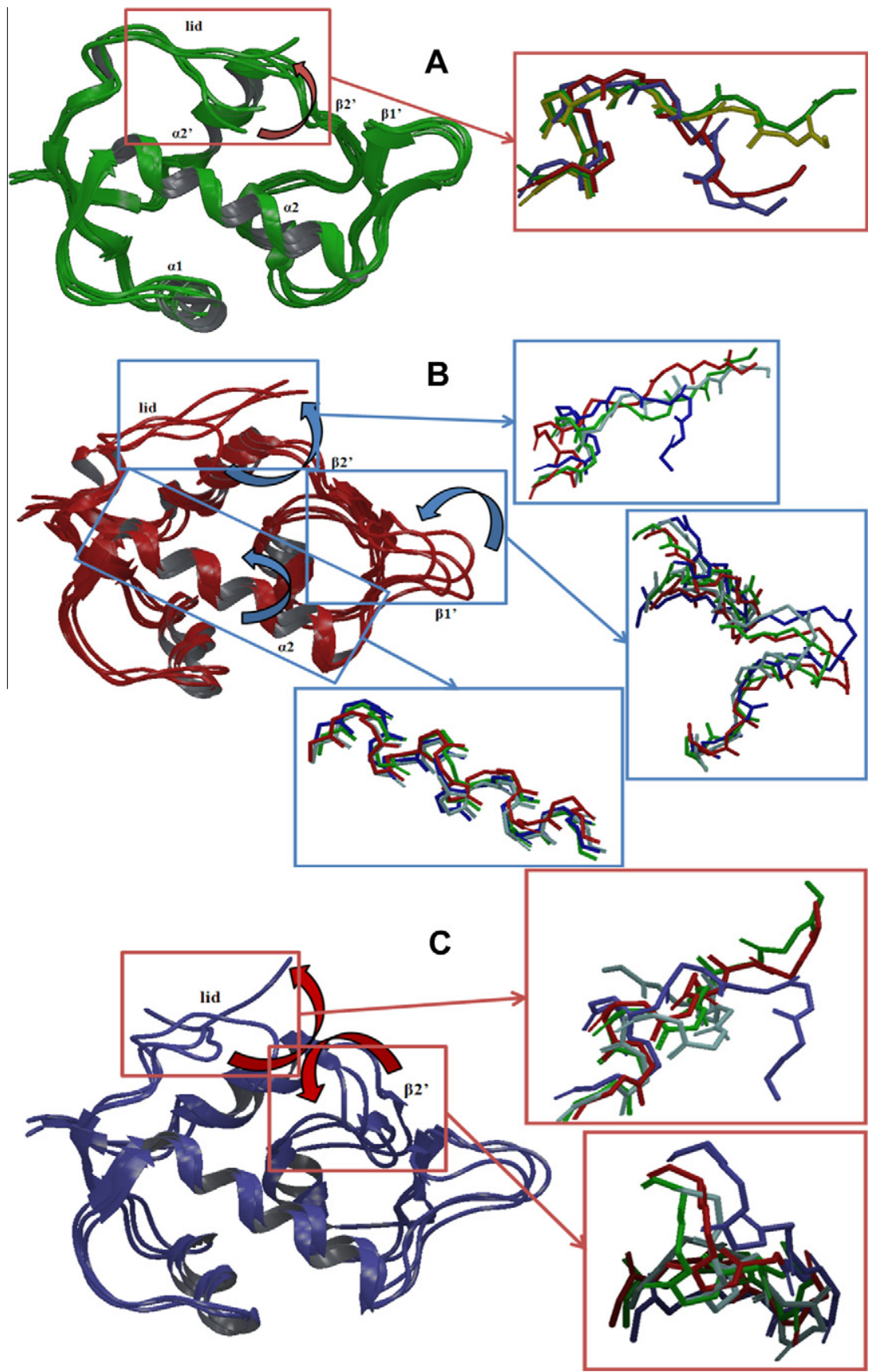

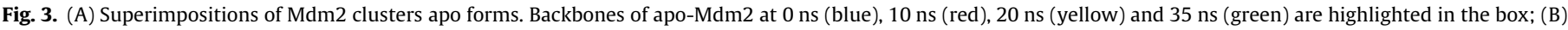

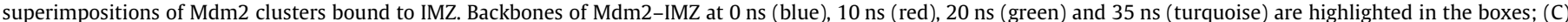

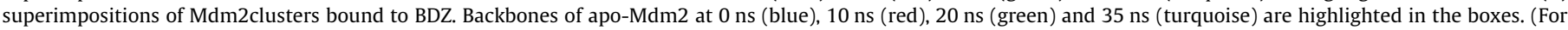
interpretation of the references to color in this figure legend, the reader is referred to the web version of this article.) 
$\beta 1^{\prime}$. There were a few residues that showed larger degree of flexibility throughout the $35 \mathrm{~ns}$ simulation (RMSF $>1.3 \AA$ ) in the region including amino acids 78-81. When comparing the mobility of apo-Mdm2 to that observed for Mdm2 bound to IMZ, a very similar trend of RMSF was observed. The mobility of Mdm2 bound to IMZ is lower than apo-Mdm2, except for residues 61-74, in proximity of the amino acids which constitute the hydrophobic binding cleft (Met62, Tyr67, Val75). The residues that showed the highest flexibility were Lys70, Ser78, and Asn79 (RMSF about $1.5 \AA$ ), located around the binding cleft. Residues which constitute binding cleft showed higher degree of flexibility. The higher RMSFs highlight the ability to better adapt itself to ligand (BDZ, IMZ), and in particular benzodiazepine core seems to make the binding pocket much more adaptive than imidazoline one.

To qualitatively analyze the conformational changes experienced by the protein, the superimposition of three clusters of structures was analyzed (Fig. 3).

Considering apo-Mdm2 superimpositions, we observed that the lid was located just above the binding cleft in the first $13 \mathrm{~ns}$. Between 13 and 19 ns, the lid displayed a movement outwards, opening the binding site. In Mdm2-IMZ system, we observed that the lid displayed a high degree of flexibility and a large movement away from the cleft. The helix $\alpha 2$ experienced an inward movement toward the center of the cleft. Furthermore, sheets $\beta 1^{\prime}-\beta 2^{\prime}$ showed a movement toward the center of the binding cleft, leading to a more closed conformation of the binding pocket. Similarly, in the case of Mdm2-BDZ superimposition, the helix $\alpha 2^{\prime}$ experienced an inward movement toward the center of mass of the cleft. The hinge that connected helix $\alpha 2^{\prime}$ with sheets $\beta 2^{\prime}$, the floor of the cleft, showed a large movement toward the center of the binding cleft. This inward movement was facilitated by the twisting motion of the hinge formed by sheets $\beta 1^{\prime}$ and $\beta 2^{\prime}$. The shape of the binding cleft changes and the cleft movement is influenced by the movement of hinges and helices located around.

Another interesting feature observed in our simulations was the dynamics of the side chains of key residues at the binding pocket, Leu54, Leu57, Ile61, Met62, Tyr67, Val75, Phe86, Phe91, Val93, Ile99, and Ile103. Leu57 is a residue that interacts with p53 via the formation of a hydrogen bond between the N $\varepsilon$ from Trp23 (p53) and the backbone carbonyl of Mdm2. When Mdm2 is unbound (Fig. 4A), Leu57 displayed rapid transitions going from $-170^{\circ}$ to $+170^{\circ}$. No significant differences were observed in the type of motions displayed by Met62, Tyr67, Val75, Phe86, and Leu54. Also for Phe91 and Val93 no significant differences in the type of motions were observed for the first $23 \mathrm{~ns}$. After that point they displayed rapid transitions.

In the case of Mdmd2 bound to BDZ (Fig. 4B) no significant differences were observed in the type of motions displayed by Leu54, except rapid transitions going from $-60^{\circ}$ to $+164^{\circ}$ and from $-145^{\circ}$ to $+178^{\circ}$ after 16 and $26 \mathrm{~ns}$, respectively. A similar situation was noticed for Leu57, which displayed transition movements in the range from $-160^{\circ}$ to $+170^{\circ}$, and for Met62. No significant differences were observed in the type of motions displayed by Tyr67 and Phe86. Val75 displayed rapid transitions going from $-170^{\circ}$ to +177 and similar situation was noticed for Phe91 and Val93. Comparing the Mdm2-IMZ librational motions to those found in the case of Mdm2-BDZ, a slight different behavior was observed (Fig. 4C). Leu54, Leu57, Val75 and Val93 displayed transition movements in the range from $-180^{\circ}$ to $+170^{\circ}$, whereas no remarkable differences were observed in the type of motions displayed by Tyr67, Phe86 and Phe91. The binding of two different ligands (IMZ and BDZ) to the cleft requires specific side-chain movements and local arrangements of the cleft. Side-chain fluctuations of the amino acids located at the binding cleft allow the binding cleft to be more flexible and to adapt itself to incoming ligand.
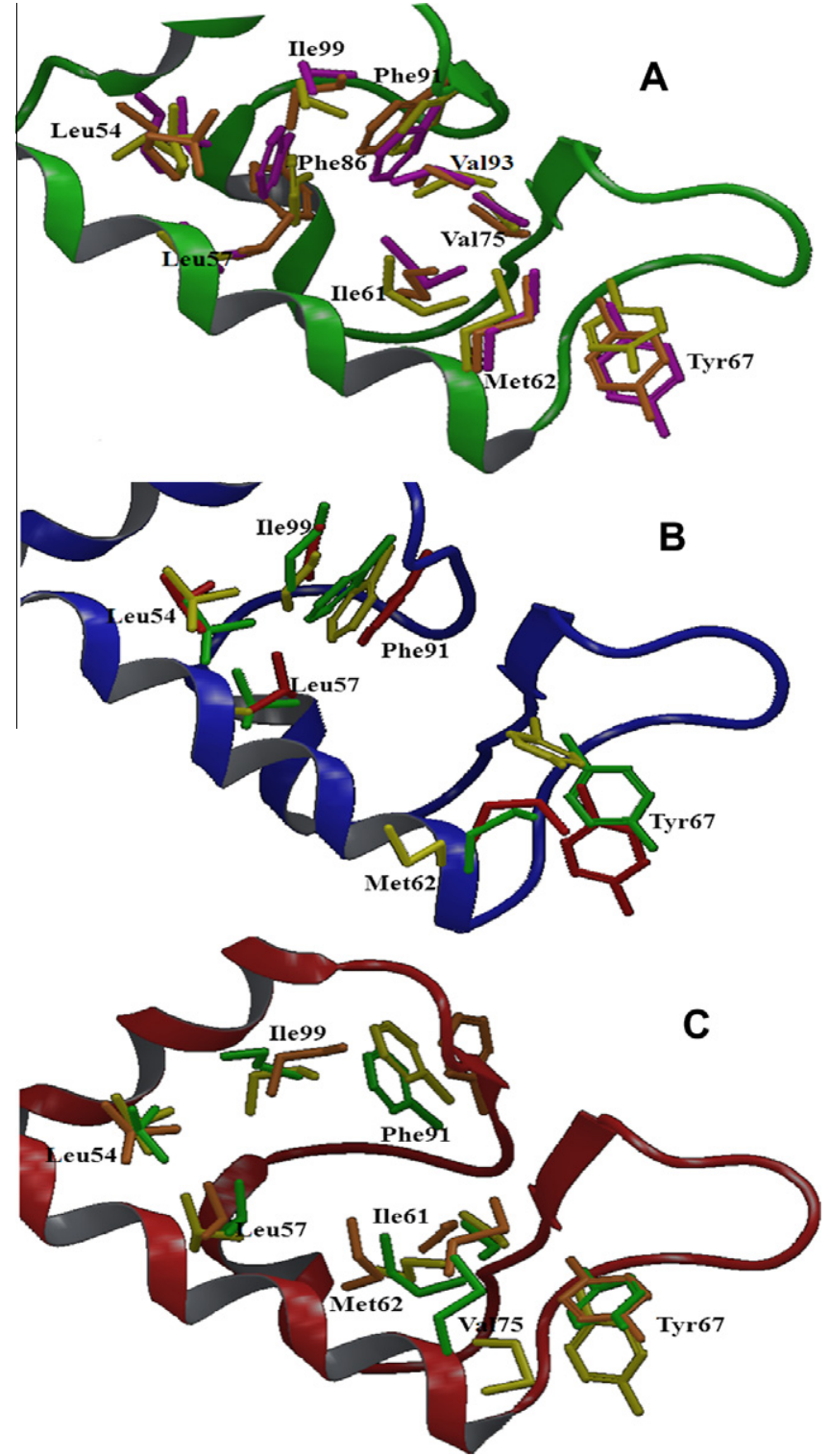

Fig. 4. (A) Side-chain motions of Mdm2 apo forms at $10 \mathrm{~ns}$ (yellow), $20 \mathrm{~ns}$ (orange), $30 \mathrm{~ns}$ (purple). (B) Side-chain motions of Mdm2 bound to BDZ at $10 \mathrm{~ns}$ (yellow), $20 \mathrm{~ns}$ (green), $30 \mathrm{~ns}$ (red). (C) Side-chain motions of Mdm2 bound to IMZ at $10 \mathrm{~ns}$ (yellow), 20 ns (orange), $30 \mathrm{~ns}$ (green). (For interpretation of the references to color in this figure legend, the reader is referred to the web version of this article.)

To investigate the binding mode of the two inhibitors, we analyzed the structure of the snapshot of Mdm2 bound to the two different compounds (IMZ and BDZ) obtained after 35 ns simulations. When the Mdm2-IMZ at 0 ns was analyzed, IMZ interacts with Gln72 through the hydroxyethyl group, which donates a hydrogen bond to the side-chain oxygen of Gln72. We observed that one bromophenyl group established non polar interaction with Leu54, the other bromophenyl group with Leu57, Phe86, Phe91, Ile99, and Ile103. In addition the hydroxyethyl side chain of IMZ established non polar interaction with Tyr67 and Ile61. When the protein-inhibitor complex, as obtained after $35 \mathrm{~ns}$ MD, was analyzed, we found that bromophenyl groups established non polar interaction with Leu57, Gly58, Ile61, Val93 and His96. The hydroxyethyl group of the IMZ formed non polar interaction with Gln72, the piperazine group with Tyr67, and 2-ethoxy-4-methoxyphenyl group with His73 and Ala43. 
In the other investigated complex, the BDZ occupies the same pockets of the peptide $\mathrm{p} 53$ side chains, and the Mdm2 interactions with the inhibitor were largely nonspecific van der Waals contacts. The BDZ pendant groups orient themselves to mimic the position of the hydrophobic side chains on one face of the helical p53 peptide ligand and thus act as an $\alpha$-helix mimetic, with one chlorophenyl moiety sitting in the Leu pocket, the other occupying the Trp pocket, and the 7-iodo-2,5-dioxo-1,2,3,5-tetrahydrobenzodiazepine moiety laying in the Phe pocket. When the protein-inhibitor complex, as obtained after 35 ns MD, was analyzed, we found that chlorophenyl groups established non polar interaction with Val93, Lys94, His96, Tyr100 and Ile103, the core dioxobenzodiazepine ring formed non polar interaction with Ile61, Tyr67, Lys94 and Gln72. These findings are partially in agreement with the data reported in Ref. [27], in which the authors performed a LDA with the aim of identifying specific residues whose conformational changes are the marker of the apo and p53-bound states of Mdm2 and MdmX.

In IMZ-Mdm2 two residues are identified as involved in the shape arrangement of the p53 binding site: Gln72 and His73. Gln72 and Tyr100 are identified in BDZ-Mdm2. Other residues are involved in the shape arrangement of the binding site, namely Ala43, Leu57, Gly58, Ile61, Tyr67, His73, Val93, Lys94, His96, and Ile103.

Therefore ligands make extensive van der Waals contacts with residues in the peptide binding cleft comprised of the structural elements $\alpha 2, \beta 1^{\prime}$, and $\alpha 2^{\prime}$. These residues are: Leu54, Leu57, Ile61 and Met62 in helix $\alpha 2$; Tyr67, Phe91, and Val93 in $\beta 1^{\prime}$ sheet; and His96 and Tyr100 in helix $\alpha 2^{\prime}$. These interactions support the observed changes in the backbone residues known to be in direct contact with the ligands. Both ligands caused similar changes in the peptide binding cleft. It was observed that, in our Mdm2-IMZ simulation, Leu57, Ile61 and Met62 (in helix a2) showed an inward movement toward the center of the binding cleft. On the contrary Phe91 showed an outward movement of about $1.5 \AA$. The wider difference was found in the case of Phe86, that showed an inward movement of about $3 \AA$.

In Mdm2-BDZ simulation Ile61, Met62, Tyr67 and Phe91 showed an inward movement toward the center of the binding cleft. The larger differences were in His96 and Val93, that showed an inward movement of about $2 \AA$. The main conformational changes were found in linker turn regions or in the $\beta$ sheets. These conformational changes seem to be sufficient to accommodate ligands that differ in their length: the changes alter the size of the cleft and indirectly affect the angle of the bottom $\alpha$ helices with respect to the cleft. The analysis of the results obtained by molecular dynamics of apo system showed a stable and less flexible structure, with the lid closing the binding cleft of the protein and leading to a closed conformation of the cleft.

Therefore, as evidenced in this study, upon binding of different ligands a rearrangement and an outward expansion of the Mdm2 helices, surrounding the binding cleft in bound systems, was observed. Ligand IMZ caused the most prominent changes in the $\beta$ sheets surrounding the binding cleft $\left(\beta 2^{\prime}\right)$, whereas BDZ produced its most significant changes in the antiparallel $\beta$-sheets, the linker regions between the $\beta$-sheets and the $\alpha$-helices that form the bottom and side walls of the cleft.

It seems that the Mdm2 domain has an intrinsic flexibility that enables it to adapt its conformation to ligands. The conformational changes alter the size of the cleft and were mainly in the linker regions suggesting that the overall dynamic nature of Mdm2 is related to dynamic movements in these regions. NMR spectroscopy studies [25] confirmed the observation that changes in chemical shift were mainly in the linker regions between the secondary structure elements and suggests that the overall dynamic nature of Mdm2 is related to dynamic movements in these regions, which can occur without any loss of the secondary structure scaffold.

\section{Appendix A. Supplementary data}

Supplementary data associated with this article can be found, in the online version, at http://dx.doi.org/10.1016/j.bbrc.2012.06.138.

\section{References}

[1] M.B. Kastan, O. Onyekwere, D. Sidransky, et al., Participation of p53 pProtein in the cellular response to DNA damage, Cancer Res. 51 (1991) 6304-6311.

[2] X. Wu, A.J. Levine, P53 and E2F-1 cooperate to mediate apoptosis, Proc. Natl. Acad. Sci. USA 91 (1994) 3602-3606.

[3] H. Hermeking, D. Eick, Mediation of c-myc induced apoptosis by p53, Science 265 (1994) 2091-2093.

[4] J.D. Oliner, J.A. Pietenpol, B. Vogelstein, et al., Oncoprotein MDM2 conceals the activation domain of tumor suppressor p53, Nature 362 (1993) 857-860.

[5] M.S. Greenblatt, W.P. Bennett, M. Hollstein, C.C. Harris, Mutations in the p53 tumor suppressor gene: clues to cancer etiology and molecular pathogenesis, Cancer Res. 54 (1994) 4855-4878.

[6] J.D. Oliner, K.W. Kinzler, B. Vogelstein, et al., Amplification of a gene encoding a p53-associated protein in human sarcomas, Nature 358 (1992) 80-83.

[7] C.E. Bueso-Ramos, Y. Yang, M. Albitar, et al., The human MDM-2 oncogene is overexpressed in leukemias, Blood 82 (1993) 2617-2623.

[8] C. Wasylyk, R. Salvi, B. Wasylyk, et al., P53 mediated death of cells overexpressing MDM2 by an inhibitor of MDM2 interaction with p53, Oncogene 18 (1999) 1921-1934.

[9] A. Bottger, V. Bottger, D.P. Lane, et al., Molecular characterization of the hdm2p53 interaction, J. Mol. Biol. 269 (1997) 744-756.

[10] C. Garcia-Echeverria, P. Chene, M.J. Blommers, P. Furet, Discovery of potent antagonists of the interaction between human double minute 2 and tumor suppressor p53, J. Med. Chem. 43 (2000) 3205-3208.

[11] T.V. Lyubomir, MDM2 inhibitors for cancer therapy, Trends Mol. Med. 13 (2007) 23-31.

[12] I. Massova, P.A. Kollman, Computational alanine scanning to probe protein-protein interactions: a novel approach to evaluate binding free energies, J. Am. Chem. Soc. 121 (1999) 8133-8143.

[13] H. Zhong, H.A. Carlson, Computational studies and peptidomimetic design for the human p53-MDM2 complex, Proteins 58 (2005) 222-234.

[14] L.M. Espinoza-Fonseca, J.G. Trujillo-Ferrara, Conformational changes of the p53-binding cleft of MDM2 revealed by molecular dynamics simulations, Biopolymers 83 (2006) 365-373.

[15] A. Macchiarulo, N. Giacchè, A. Carotti, et al., Targeting the conformational transitions of MDM2 and MDMX: insights into dissimilarities and similarities of p53 recognition, J. Chem. Inf. Model. 48 (2008) 1999-2009.

[16] S.A. Showalter, L. Bruschweiler-Li, R. Bruschweiler, et al., Quantitative lid dynamics of MDM2 reveals differential ligand binding modes of the p53binding cleft, J. Am. Chem. Soc. 130 (2008) 6472-6478.

[17] T.L. Joseph, A. Madhumalar, C. Verma, et al., Differential binding of p53 and nutlin to MDM2 and MDMX: computational studies, Cell Cycle 9 (2010) 11671181 .

[18] G.W. Yu, M. Vaysburd, A.R. Fersht, et al., Structure of human MDM4 N-terminal domain bound to a single-domain antibody, J. Mol. Biol. 385 (2009) 15781589.

[19] S.G. Dastidar, A. Madhumalar, C.S. Verma, et al., Forces mediating proteinprotein interactions: a computational study of p53 "approaching" MDM2, Theor. Chem. Acc. 125 (2010) 621-635.

[20] H.J.C. Berendsen, D. Van der Spoel, R. Van Drunen, GROMACS: a messagepassing parallel molecular dynamics implementation, Comp. Phys. Comm. 91 (1995) 43-56.

[21] E. Lindahl, B. Hess, D. Van der Spoel, GROMACS 3.0: a package for molecular simulation and trajectory analysis, J. Mol. Mod. 7 (2001) 306-317.

[22] A. Pedretti, L. Villa, G. Vistoli, VEGA: a versatile program to convert, handle and visualize molecular structure on Windows-based PCs, J. Mol. Graph. Model. 21 (2002) 47-49.

[23] W. Humphrey, A. Dalke, K. Schulten, VMD: visual molecular dynamics, J. Mol. Graph. Model 14 (1996) 33-38.

[24] Schrödinger Suite 2008 Induced Fit Docking protocol; Glide version 5.0, Schrödinger, LLC, New York, NY, 2005; Prime version 1.7, Schrödinger, LLC, New York, NY, 2005.

[25] D.C. Fry, S.D. Emerson, F. Podlaski, et al., NMR structure of a complex between MDM2 and a small molecule inhibitor, J. Biomol. NMR 30 (2004) 163-173.

[26] O. Schon, A. Friedler, S. Freund, A.R. Fersht, Binding of p53-derived ligands to MDM2 induces a variety of long range conformational changes, J. Mol. Biol. 336 (2004) 197-202.

[27] A. Carotti, A. Macchiarulo, N. Giacchè, R. Pellicciari, Targeting the conformational transitions of MDM2 and MDMX: insights into key residues affecting p53 recognition, Proteins 77 (2009) 524-535. 